\title{
Rare Though Not Mutually Exclusive: A Report of Three Cases of Concomitant KRAS and BRAF Mutation and a Review of the Literature
}

\author{
Ibrahim Halil Sahin, Syed M.A. Kazmi, Jeffrey T. Yorio, Nishin A. Bhadkamkar, Bryan K. Kee, Christo- \\ pher R. Garrett ${ }^{\bowtie}$
}

Department of Gastrointestinal Medical Oncology, The University of Texas M.D. Anderson Cancer Center, Unit 426, 1515 Holcombe Boulevard, Houston, Texas, 77030-4009.

$\triangle$ Corresponding author: Christopher Garrett, MD, Department of Gastrointestinal Medical Oncology, MD Anderson Cancer Center, Unit 426, 1515 Holcombe Boulevard, Houston, Texas, 77030-4009. Email: cgarrett@mdanderson.org Tel: 713-792-2828 Fax: 713-792-4032.

(C) Ivyspring International Publisher. This is an open-access article distributed under the terms of the Creative Commons License (http://creativecommons.org/ licenses/by-nc-nd/3.0/). Reproduction is permitted for personal, noncommercial use, provided that the article is in whole, unmodified, and properly cited.

Received: 2012.12.18; Accepted: 2013.02.07; Published: 2013.03.28

\begin{abstract}
KRAS mutations occur frequently in colorectal cancers (CRC) and predict lack of response to anti-epidermal growth factor receptor (EGFR) monoclonal antibody therapy. CRC BRAF mutations, most commonly at $\mathrm{V} 600 \mathrm{E}$, occur less than $10 \%$ of the time, and occur usually in KRAS wild-type tumors, and more frequently in microsatellite instable tumors. Concomitant KRAS and BRAF mutant CRCs are rare (occurring in $0.001 \%$ ); BRAF mutations should not be routinely tested in patients with KRAS mutant tumors, unless the patients is participating in a clinical trial enriching for the presence of a KRAS or BRAF tumor. Clinical trials treating patients with either KRAS or BRAF mutant tumors should address eligibility of patients with concomitant KRAS and BRAF mutations.
\end{abstract}

Key words: Concomitant KRAS, BRAF

\section{Introduction}

Mutations in codons 12 and 13 of Kirsten rat sarcoma viral oncogene homolog (KRAS) and b-Raf murine sarcoma viral oncogene homolog B1 (BRAF) genes are frequently present in tumors of patients with metastatic colorectal cancer (CRC); they are observed in $30-45 \%$ and $5-20 \%$ of cases respectively [1] . Less commonly detected CRC tumor mutations are codon 61,146 of the KRAS oncogene, and at other sites in the BRAF gene. KRAS and BRAF mutations have been frequently described as mutually exclusive in CRC, in that tumors usually have neither, or only one, of these specific mutations. Rarely patients may have CRC with coincident KRAS and BRAF mutations; herein we report three such cases and review of the literature. Although highly uncommon, identifi- cation of this dual tumor genotype is important, as current clinical trials are being designed enriching for patients whose tumors harbor either a KRAS or BRAF mutation, but haven't addressed those patients with coincident KRAS and BRAF mutations. Due to the infrequent rate of coincident mutation, it is not known if these patients have a tumor biology distinct from KRAS or BRAF mutant tumors.

\section{Method and Case Presentations}

Under an Institutional Review Board approved retrospective chart review protocol (DR-11-0113), we reviewed samples tested at a clinical laboratory for tumor KRAS and/or BRAF mutations from 2008-2011. All specimens were ordered by a clinician and deter- 
mined to be medically indicated. DNA was extracted from microdissected paraffin-embedded tumor and analyzed by a polymerase chain reaction (PCR)-based DNA sequencing method to examine codons 12, 13 and 61 of the KRAS proto-oncogene. The sensitivity of detection of this assay is approximately 1 in 10 mutation-bearing cells in microdissected area. DNA was also analyzed by PCR and a pyrosequencing method for codons 595 to 600 of exon 16 of BRAF oncogene; the methods for these assays have been described elsewhere [2]. A total 6,633 CRC patient tumors were screened for mutation analyses and of those 1,483 case were tested for both KRAS and BRAF mutations. 644 were KRAS-mutant $(43 \%)$ and $3(0.2 \%)$ of these were bearing a concomitant BRAF (V600E) mutation. The first patient is a 65 year-old woman who presented with small bowel obstruction. A transverse colon mass was found and resected. The patient underwent a biopsy of the metastatic liver lesion that was sent for KRAS mutation and BRAF mutation analysis; a tumor mutation was detected in codon 12 (GGT to GAT) of the KRAS gene that would change the encoding amino acid from glycine to aspartic acid (G12D) and codon 600 (GTG to GAG) in exon 15 of the BRAF gene that would change the encoding amino acid from valine to glutamine (V600E). The second case identified was a 72 year-old woman with rectal cancer metastatic to the lungs; similar tumor mutations were detected in KRAS (G12D) and BRAF (V600E) proto-oncogenes. In both cases the tumors were microsatellite stable; concomitant BRAF and KRAS mutations were not identified among patients samples' whose tumors were microsatellite instable. The third case is 51 year-old man who presented with abdominal pain and was found to have a descending colon cancer with liver metastasis. In specimen from liver lesion, KRAS (G12D) and BRAF (V600E) mutations were detected. Patient was not tested for microsatellite stability.

Table I. Large Studies of KRAS and BRAF Tumor Genotyping in Patients with Colorectal Cancer.

\begin{tabular}{llll}
\hline Author & $\begin{array}{l}\text { KRAS mutant/Total } \\
\text { (percentage) }\end{array}$ & $\begin{array}{l}\text { BRAF mutant } \\
\text { (percentage) }\end{array}$ & $\begin{array}{l}\text { Concomitant } \\
\text { BRAF and KRAS mutations }\end{array}$ \\
\hline VanCutsem [5] & $397 / 1,063(37.4 \%)$ & $60 / 999(6.0 \%)$ & $1 / 999(0.1 \%)$ \\
Lamy [6] & $450 / 1077(41.8 \%)$ & $26 / 397(6.5 \%)$ & $0 / 397(0 \%)$ \\
Price [7] & $90 / 315(28.8 \%)$ & $33 / 315(10.6 \%)$ & $0 / 315(0 \%)$ \\
Maughan [8] & $565 / 1294(43.7 \%)$ & $102 / 1189(8.5 \%)$ & $0 / 1189(0 \%)$ \\
Li [9] & $63 / 200(31.5 \%)$ & $14 / 200(6.5 \%)$ & $0 / 200(0 \%)$ \\
Bokemeyer [10] & $136 / 315(43.2 \%)$ & $11 / 309(3.6 \%)$ & $0 / 309(0 \%)$ \\
DeRoock [11] & $299 / 747(40.0 \%)$ & $36 / 761(4.7 \%)$ & $0 / 761(0 \%)$ \\
Total & $\mathbf{2 1 1 6 / 5 2 8 7 ( 4 0 . 0 \% )}$ & $\mathbf{2 9 7 / 4 3 2 3 ( 6 . 9 \% )}$ & $\mathbf{1 / 4 3 2 3 ( \mathbf { 0 . 0 2 } \% )}$ \\
\hline
\end{tabular}

\section{Discussion}

Coincident KRAS and BRAF mutations appear to be rare entity (present in 3 out of over 1,928 samples tested). Due to the infrequent observation of this phenomena, it is not clear whether or not these tumors have a different biology and natural history than KRAS or BRAF mutant tumors, or which of the two mutations is the dominant oncogene driving tumor proliferation. A prior study has demonstrated that concomitant tumor mutations have been relatively frequently observed human CRC cell line; in this study, of 24 human CRC cell lines, one cell line (GP2D) demonstrated mutations in both KRAS and BRAF oncogenes (however the BRAF mutation was in the less frequently observed at the codon 529 site) [3]. In an analysis of 250 patients tumor specimens with microsatellite stable disease, KRAS mutations were identified in $45.2 \%$ of primary tumors, and that concomitant KRAS and BRAF mutations were found in 10 lymph nodes (35.7\%) from a total 28 samples whereas only 3 of primary tumor with negative lymph nodes were positive for coincident mutation $(2.3 \%)$ [4]. There was also noted to be a higher proportion of concomitant mutations based on the degree of transmural penetration of the tumor; $1 / 36(2.8 \%)$ for T2 tumors and $3 / 32$ (9.4\%) for T4 tumors, suggesting activation of both genes is associated with progression of disease.

Since KRAS mutation testing became standard-of-care for determining lack of treatment efficacy with antibody anti-epidermal growth factor (EGFR) monoclonal antibody therapy in patients with advanced $C R C$, large studies involving KRAS and BRAF mutation analysis CRC screenings have been undertaken; the results of studies with over 200 patients is demonstrated in Table 1. Unlike study by Olivera et al [4], all of these large data sets demonstrated that in CRC KRAS and BRAF mutations were mutually exclusive, except in one case [5]. In one other series, two patients had tumors with two distinct KRAS mutations [6]. 
Molecular profiling demonstrates that KRAS and BRAF mutant tumors have very different gene signatures suggesting different signaling pathways are activated [12]. In addition early data suggest outcomes with the use of anti-EGFR monoclonal antibody therapy appear to be different depending on the site of KRAS mutation [13]. At this time it is not known which gene expression profile pattern of concomitant KRAS and BRAF tumors is, and whether it more represents a KRAS tumor gene signature or BRAF signature. Given fact of tumor heterogeneity and the large variety of mutations observed in this disease, as well as epigenetic changes observed, it is likely that in the future, tumor gene expression profiling will provide a better indication of the activation of tumor signaling, and be a better biomarker of treatment efficacy.

Strengths of our study are screened large patient population and available detailed clinical and pathological information. The main limitation, however, were retrospective nature of study and possible artefactual mutation detection. Further multi-center prospective studies are necessary to understand true frequency and the role concomitant KRAS and BRAF mutations.

\section{Conclusions}

Concomitant KRAS and BRAF tumor mutations are rare enough to be considered virtually (albeit not entirely) mutually exclusive. Since KRAS and microsatellite instability analysis are the only validated negative biomarkers of therapy efficacy in CRC, routine analysis for BRAF mutations in KRAS wild type tumors is not recommended. However if patients are being considered for clinical trial, especially those in which the eligibility require the presence of a KRAS mutation, BRAF mutation analysis would be highly advisable. Future enriched clinical trials for KRAS or BRAF should specifically address eligibility of patients whose tumors harbor a concomitant KRAS and BRAF mutation.

\section{Competing Interests}

The authors have declared that no competing interest exists.

\section{References}

1. Lin JS, Webber EM, Senger CA, Holmes RS, Whitlock EP. Systematic review of pharmacogenetic testing for predicting clinical benefit to anti-EGFR therapy in metastatic colorectal cancer. American journal of cancer research. 2011; 1: 650-62.

2. Lang AH, Drexel H, Geller-Rhomberg S, Stark N, Winder T, Geiger K, et al. Optimized allele-specific real-time PCR assays for the detection of common mutations in KRAS and BRAF. The Journal of molecular diagnostics : JMD. 2011; 13: 23-8. doi:10.1016/j.jmoldx.2010.11.007.

3. Seth R, Crook S, Ibrahem S, Fadhil W, Jackson D, Ilyas M. Concomitant mutations and splice variants in KRAS and BRAF demonstrate complex perturbation of the Ras/Raf signalling pathway in advanced colorectal cancer. Gut. 2009; 58: 1234-41. doi:10.1136/gut.2008.159137.

4. Oliveira C, Velho S, Moutinho C, Ferreira A, Preto A, Domingo E, et al. KRAS and BRAF oncogenic mutations in MSS colorectal carcinoma progression. Oncogene. 2007; 26: 158-63. doi:10.1038/sj.onc.1209758.

5. Van Cutsem E, Kohne CH, Lang I, Folprecht G, Nowacki MP, Cascinu S, et al. Cetuximab plus irinotecan, fluorouracil, and leucovorin as first-line treatment for metastatic colorectal cancer: updated analysis of overall survival according to tumor KRAS and BRAF mutation status. Journal of clinical oncology : official journal of the American Society of Clinical Oncology. 2011; 29: 2011-9. doi:10.1200/JCO.2010.33.5091.

6. Lamy A, Blanchard F, Le Pessot F, Sesboue R, Di Fiore F, Bossut J, et al. Metastatic colorectal cancer KRAS genotyping in routine practice: results and pitfalls. Modern pathology : an official journal of the United States and Canadian Academy of Pathology, Inc. 2011; 24: 1090-100. doi:10.1038/modpathol.2011.60.

7. Price TJ, Hardingham JE, Lee CK, Weickhardt A, Townsend AR, Wrin JW, et al. Impact of KRAS and BRAF Gene Mutation Status on Outcomes From the Phase III AGITG MAX Trial of Capecitabine Alone or in Combination With Bevacizumab and Mitomycin in Advanced Colorectal Cancer. Journal of clinical oncology : official journal of the American Society of Clinical Oncology. 2011; 29: 2675-82. doi:10.1200/JCO.2010.34.5520.

8. Maughan TS, Adams RA, Smith CG, Meade AM, Seymour MT, Wilson $\mathrm{RH}$, et al. Addition of cetuximab to oxaliplatin-based first-line combination chemotherapy for treatment of advanced colorectal cancer: results of the randomised phase 3 MRC COIN trial. Lancet. 2011; 377: 2103-14. doi:10.1016/S0140-6736(11)60613-2.

9. Li HT, Lu YY, An YX, Wang X, Zhao QC. KRAS, BRAF and PIK3CA mutations in human colorectal cancer: relationship with metastatic colorectal cancer. Oncology reports. 2011; 25: 1691-7. doi:10.3892/or.2011.1217.

10. Bokemeyer C, Bondarenko I, Hartmann JT, de Braud F, Schuch G, Zubel A, et al. Efficacy according to biomarker status of cetuximab plus FOLFOX-4 as first-line treatment for metastatic colorectal cancer: the OPUS study. Annals of oncology : official journal of the European Society for Medical Oncology / ESMO. 2011; 22: 1535-46. doi:10.1093/annonc/mdq632.

11. De Roock W, Claes B, Bernasconi D, De Schutter J, Biesmans B, Fountzilas G, et al. Effects of KRAS, BRAF, NRAS, and PIK3CA mutations on the efficacy of cetuximab plus chemotherapy in chemotherapy-refractory metastatic colorectal cancer: a retrospective consortium analysis. Lancet Oncol. 2010; 11: 753-62. doi:10.1016/S1470-2045(10)70130-3.

12. Tejpar S, Popovici V, Dolarenzi M, Budinska E, Estrella H, Mao M, et al. Mutant KRAS and BRAF gene expression profiles in colorectal cancer: Results of the translational study on the PETACC 3-EORTC 40993-SAKK 60-00 trial. Journal of clinical oncology: official journal of the American Society of Clinical Oncology. 2010; 28:15s.

13. De Roock W, Jonker DJ, Di Nicolantonio F, Sartore-Bianchi A, Tu D, Siena S, et al. Association of KRAS p.G13D mutation with outcome in patients with chemotherapy-refractory metastatic colorectal cancer treated with cetuximab. JAMA : the journal of the American Medical Association. 2010; 304: 1812-20. doi:10.1001/jama.2010.1535. 